\title{
Temporal $v_{\mathrm{P}} / v_{\mathrm{S}}$ variation characteristics in different zones of China's Capital Circle area before and after Wen'an earthquake*
}

\author{
WANG Lin-ying ${ }^{1), *}$ (王林瑛) GUO Yong-xia ${ }^{1)}$ (郭永霞) $\quad$ LIU Fang $^{2)}$ (刘 芳) \\ JIANG Chang-sheng ${ }^{1)}$ (蒋长胜) \\ 1) Institute of Geophysics, China Earthquake Administration, Beijing 100081, China \\ 2) Earthquake Administration of Inner Mongolia Autonomous Region, Hohhot 010051, China
}

\begin{abstract}
On July 4, 2006, an earthquake of $M_{\mathrm{S}} 5.1$ took place in Wen'an, Hebei Province, just at the south center of China's Capital Circle area digital seismograph network. It is the strongest event recorded ever since the network went into operation in 2002. We processed the vast amounts of phase data yielded by the 107 digital seismic stations between 2002 2007 using Wadati method. In order to improve the precision and stability of shear and compressional wave velocities $\left(v_{\mathrm{p}} / v_{\mathrm{S}}\right)$ calculation, we impose a number of restrictions on the computation environment and condition, e.g., the earthquakes are densely concentrated, selected stations are limited in range, the number of stations involved in the computation is larger than 5 and linear fitting features high precision and small error. Under these restrictions, the study shows that $v_{\mathrm{P}} / v_{\mathrm{S}}$ in and around Wen'an and Tangshan underwent a normal-low-normal process one year before Wen'an earthquake, $v_{\mathrm{P}} / v_{\mathrm{S}}$ became obviously low and the low ratio lasted for about one year, meanwhile, little variation of $v_{\mathrm{P}} / v_{\mathrm{S}}$ was seen in Xingtai, northwest of Beijing, southwest of Beijing, Beijing-Tianjin and Beijing; after the quake, the $v_{\mathrm{P}} / v_{\mathrm{S}}$ returned normal in Wen'an and Tangshan. Error and stability analysis of the calculated result for $v_{\mathrm{P}} / v_{\mathrm{S}}$ shows it is convincible that anomaly appeared in and around Wen'an and Tangshan before Wen'an earthquake.
\end{abstract}

Key words: Wen'an earthquake; ratio of shear and compressional wave velocities; earthquake prediction CLC number: P315.3 Document code: A

\section{Introduction}

Based on the presupposition of ideal homogeneous elastic medium from the hypocenter to the ground surface, Japanese seismologist Wadati developed the Wadati method in 1928 for directly computing $v_{\mathrm{P}} / v_{\mathrm{S}}$ from the hypocenter to the seismic stations on the ground surface by using linear relationship between direct P-wave arrival time and P-S wave arrival time difference, the ratio is taken as an important physical parameter reflecting medium property of the upper crust (LI et al, 1981). In the course of earthquake development, the physical state of the focus region would undergo a series of changes, e.g., microfracturing, dilatancy, plastic hardening and phase trans-

\footnotetext{
* Received 2007-09-26; accepted in revised form 2008-03-22

Foundation item: Special Fund for Basic Scientific Research, IGPCEA (DQJB06B03) and National Program for Key Science and Technology Projects during the 11th Five-year Plan Period (2006BAC01B03-04-04).

Contribution No.08FE3004, Institute of Geophysics, China Earthquake Administration.

^Author for correspondence: wang_linying@163.com
} 
formation, and the velocity of seismic waves would change accordingly as they pass through the focus region. This provides an important basis for predicting earthquakes with $v_{\mathrm{P}} / v_{\mathrm{S}}$ anomaly (FENG, 1981). In the early 1950s, Japanese scholar Hayakawa concluded that anomalies in seismic wave velocity ratio exist in some focus regions, and the duration is directly proportional to the magnitude of earthquakes. Earthquake experiment sites were established one after another in the former USSR, US and Japan, and anomalies in seismic wave velocity ratio before some moderate and strong earthquakes were observed in the 1960s 1970s. To explain the mechanism of the anomalies, a dilatancy model was developed by a group of scientists represented by Nur (LI, 1981). Meanwhile, the anticipated variations in seismic wave velocity ratio were not observed in the focus regions of some relatively large shocks by higher precision traveltime data of artificial earthquakes (McEvilly and Johnson, 1974; Kanamori and Fuis, 1976), and this shifted international research focus from the application of seismic velocity ratio method to the research on brittleness, rheology and meltability of media and lithologic composition (e.g., Walck, 1988; Hauksson and Haase, 1994). In recent years, with the improvement of observation condition and introduction of new techniques, the study on variation characteristics of wave velocity ratio has again attracted wide attention (Nakamura et al, 2002). The latest vibroseis experiments show that variations in velocity ratio have really been observed in the crust (Li et al, 1998; Korneev et al, 2000), and obvious variations in velocity ratio have also been observed in the focus regions before some strong earthquakes by using phase delay of the "recurrent earthquakes" in the same location (Schaff and Beroza, 2004).

Observing temporal variations of subsurface medium property is an important approach as well as a potential research field for the study of earthquake prediction. Velocity estimation of seismic waves and information acquisition concerning subsurface media property variation is absolutely significant and essential for predicting physical parameters of moderate and strong earthquakes, especially for increasing earthquake-related subsurface information. Since 1970s, Chinese scholars have published a series of significant results about the spatial distribution characteristics of $v_{\mathrm{P}} / v_{\mathrm{S}}$ in different areas and about the difference in physical property between tectonic blocks (FENG et al, 1974, 1978; FENG et al, 1976; ZHANG et al, 2004a, b; ZHAO et al, 2005; LI and ZHANG, 2004; ZHANG et al, 2007). Especially since 2004, with the constant accumulation of digital seismic data, more and more researches in China have been focused on the analysis of variations in seismic wave velocity ratio before moderate and strong earthquakes (DIAO et al, 2005; ZHANG, 2005; LI and LIU, 2006; ZHANG et al, 2006; ZOU et al, 2006). As the number of case study on $v_{\mathrm{P}} / v_{\mathrm{S}}$ variation of moderate and strong earthquakes and reservoir-induced earthquakes becomes gradually larger, the credibility of anomalies in seismic wave velocity ratio before earthquakes has been fully affirmed.

On July 4, 2006, an earthquake of $M_{\mathrm{S}} 5.1$ took place at the south center of China's Capital Circle area digital seismograph network. It is the strongest event recorded ever since the network went into operation in 2002. Based on phase data from 107 stations of the network, this study shows that $v_{\mathrm{P}} / v_{\mathrm{S}}$ underwent a normal-low-normal process before Wen'an earthquake. The objectivity and stability of the process is also discussed in this paper.

\section{Wave velocity calculation method and data processing}

Using Wadati method, we calculated $v_{\mathrm{P}} / v_{\mathrm{S}}$ for Wen'an, Beijing, Beijing-Tianjin, Tangshan, Luanxian, southwest of Beijing, northwest of Beijing and Xingtai respectively, based on 
phase-containing earthquake report of the Capital Circle area digital seismograph network between January 2002 and April, 2007 provided by the fourth division of the Institute of Geophysics, China Earthquake Administration (IGPCEA).

The method used in this study for computing $v_{\mathrm{P}} / v_{\mathrm{S}}$ is the Wadati method and the seismic data used are mostly arrival time data of direct $\mathrm{P}$ and $\mathrm{S}$ waves. For ideal homogeneous elastic media, $\mathrm{P}$-wave velocity, $v_{\mathrm{P}}$, and S-wave velocity, $v_{\mathrm{P}}$ are related to Poisson's ratio of media $\sigma$, Young's modulus $E$, elastic constant of media $\mu$ and medium density $\rho$ as follows (LI, 1981):

$$
\begin{gathered}
v_{\mathrm{P}}=\sqrt{\frac{E}{\rho}\left(1+\frac{2 \sigma^{2}}{1-\sigma-2 \sigma^{2}}\right)} \\
v_{\mathrm{S}}=\sqrt{\frac{E}{\rho} \frac{1}{2(1+\sigma)}} \\
\frac{v_{\mathrm{P}}}{v_{\mathrm{S}}}=\sqrt{\frac{2(1-\sigma)}{1-2 \sigma}}
\end{gathered}
$$

Equation (3) shows that $v_{\mathrm{P}} / v_{\mathrm{S}}$ is a function of Poisson's ratio $\sigma$, and it mainly reflects the variation in Poisson's ratio of the upper crust. In this study, we calculated P- and S-wave velocities while calculating $v_{\mathrm{P}} / v_{\mathrm{S}}$, besides, we examined variation characteristics of $\mathrm{P}$ - and S-wave velocities while analyzing anomaly in $v_{\mathrm{P}} / v_{\mathrm{S}}$ before Wen'an earthquake.

\section{Restrictions on $v_{\mathrm{p}} / v_{\mathrm{S}}$ computation condition}

The most difficult point for seismic wave velocity calculation lies in the improvement of phase identification precision and judgment of the reliability. Major factors affecting the precision of velocity calculation include precision of $\mathrm{P}$ - and S-wave arrival time identification, number of stations involved in the fitting process and precision of earthquake location. In order to minimize the effects of the above-mentioned factors, we put the following restrictions on the range of study, selection of earthquakes and precision of computation.

\subsection{Restrictions on the studied zones}

In recent years, seismologists in China and abroad have paid more attention to earthquake recurrence. One of the effective means for improving the precision of wave velocity estimation is to observe wave velocity variation of the earthquakes with relatively fixed focal depth, magnitude and similar waveform, under the condition that the elements affecting wave velocity variation are restricted to one possible factor of medium property.

By Wadati method, the assumed ideal environment is layered homogeneous media and the difference in earthquake distribution is not taken into account in the investigation, but in practical research, lateral difference in medium property between different zones does exist objectively, which is one of the important factors leading to different results of wave velocity calculation. To minimize the difference in seismic wave path while securing sample size, we made use of the fact that earthquakes occur in clusters in Tangshan, Xingtai and Datong historical earthquake regions. Variations in media property of different zones are observed carefully, with the focus on the historical regions where earthquakes are densely concentrated. For non-historical earthquake regions, we tried to limit the earthquakes chosen for study within a $1^{\circ} \times 1^{\circ}$ range, so as to reduce the effect of large difference in seismic wave path caused by sparsely distributed earthquakes and thus, improve the stability of wave velocity calculation. 


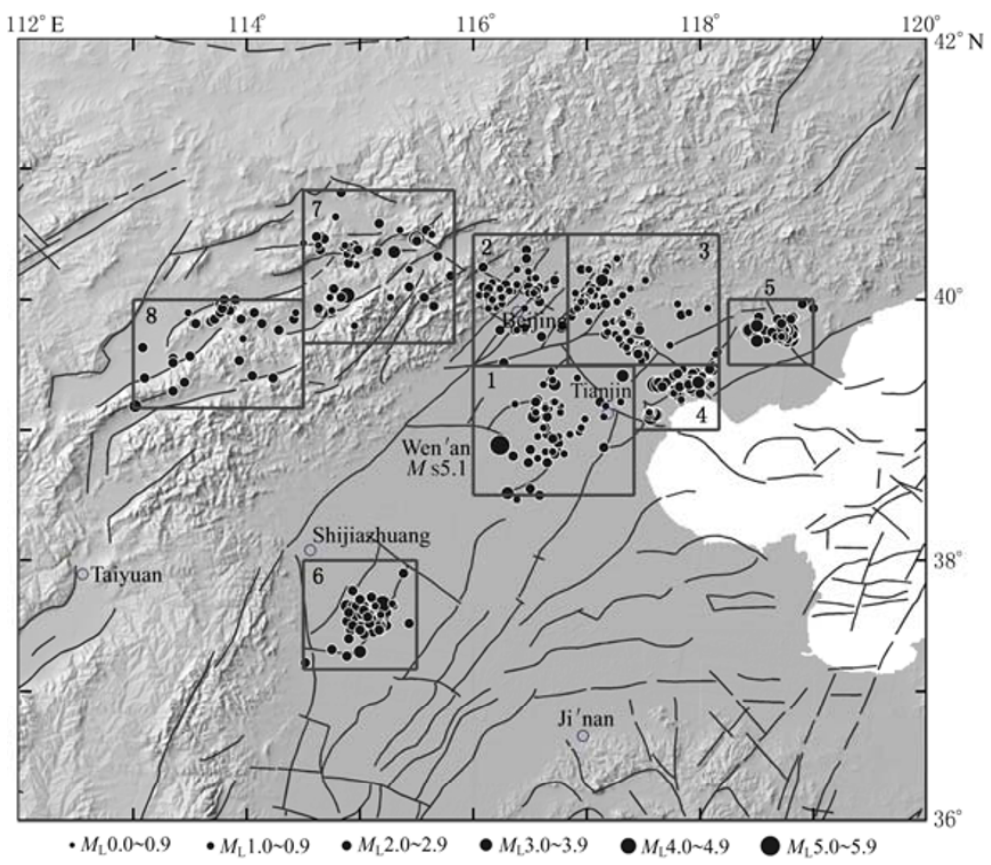

Figure 1 Spatial distribution of the eight zones for seismic study in the Capital Circle area Zone 1: Wen'an; zone 2: Beijing; zone 3: Beijing-Tianjin; zone 4: Tangshan; zone 5: Luanxian; zone 6: Xingtai; zone 7: Northwest of Beijing; zone 8: Southwest of Beijing

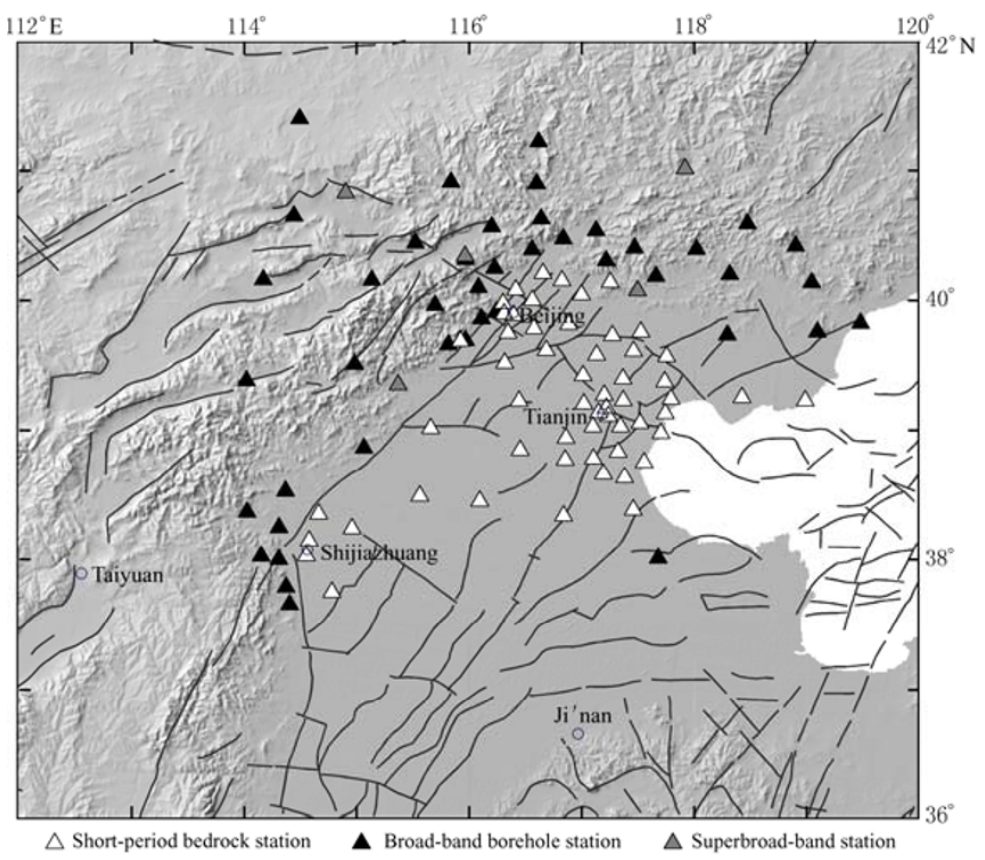

Figure 2 Distribution of stations of China's Capital Circle area digital seismograph network

To identify similarities and differences in subsurface media between different zones in the Capital circle area, we chose four earthquake cluster zones near Wen'an earthquake epicenter (Wen'an, Tangshan, Beijing-Tianjin and Beijing) and four cluster zones relatively farther away 
from the epicenter (Luanxian, Xingtai, northwest of Beijing, southwest of Beijing), for separate studies of their temporal $v_{\mathrm{P}} / v_{\mathrm{S}}$ variation characteristics. Table 1 shows the distribution range of the selected earthquakes in the cluster zones. The smallest range of a cluster zone is $30^{\prime} \times 30^{\prime}$, the largest $1^{\circ} \times 1^{\circ}$ and the spatial range is limited within $(50 \mathrm{~km} \times 50 \mathrm{~km}) \sim(100 \mathrm{~km} \times 100 \mathrm{~km})$ (Figure 1, Table 1). We chose $t_{\mathrm{S}}-t_{\mathrm{P}} \leq 25 \mathrm{~s}$ or $t_{\mathrm{S}}-t_{\mathrm{P}} \leq 20 \mathrm{~s}$, and limited the sections where seismic rays pass to a certain range. The spatial ranges of such sections for each cluster zone correspond to those shown in Table 2 and Figure 9.

Table 1 Distribution range of the selected earthquakes in the earthquake cluster zones

\begin{tabular}{clccccc}
\hline No. & \multicolumn{1}{c}{ Zone } & Time period & $\varphi_{\mathrm{N}}$ & $\lambda_{\mathrm{E}}$ & $\begin{array}{c}\text { Smallest } \\
\text { magnitude }\end{array}$ & $\begin{array}{c}\text { Total number of } \\
\text { qualified shocks }\end{array}$ \\
\hline 1 & Wen'an & $2002-01 \sim 2007-10$ & $38^{\circ} 30^{\prime} \sim 39^{\circ} 30^{\prime}$ & $116^{\circ} 00^{\prime} \sim 117^{\circ} 20^{\prime}$ & 1.0 & 45 \\
2 & Beijing & $2002-01 \sim 2007-10$ & $39^{\circ} 40^{\prime} \sim 40^{\circ} 20^{\prime}$ & $116^{\circ} 00^{\prime} \sim 116^{\circ} 50^{\prime}$ & 1.0 & 57 \\
3 & Beijing-Tianjin & $2002-01 \sim 2007-10$ & $39^{\circ} 30^{\prime} \sim 40^{\circ} 30^{\prime}$ & $116^{\circ} 50^{\prime} \sim 118^{\circ} 10^{\prime}$ & 1.0 & 86 \\
4 & Tangshan & $2002-01 \sim 2007-10$ & $39^{\circ} 00^{\prime} \sim 39^{\circ} 30^{\prime}$ & $117^{\circ} 30^{\prime} \sim 118^{\circ} 00^{\prime}$ & 1.5 & 59 \\
5 & Luanxian & $2002-01 \sim 2007-10$ & $39^{\circ} 30^{\prime} \sim 40^{\circ} 00^{\prime}$ & $118^{\circ} 10^{\prime} \sim 118^{\circ} 50^{\prime}$ & 2.0 & 109 \\
6 & Xingtai & $2002-01 \sim 2007-10$ & $37^{\circ} 20^{\prime} \sim 38^{\circ} 10^{\prime}$ & $114^{\circ} 50^{\prime} \sim 115^{\circ} 30^{\prime}$ & 2.0 & 67 \\
7 & NW of Beijing & $2002-01 \sim 2007-04$ & $39^{\circ} 40^{\prime} \sim 40^{\circ} 40^{\prime}$ & $114^{\circ} 30^{\prime} \sim 115^{\circ} 30^{\prime}$ & 2.0 & 54 \\
8 & SW of Beijing & $2002-01 \sim 2007-04$ & $39^{\circ} 10^{\prime} \sim 40^{\circ} 00^{\prime}$ & $113^{\circ} 00^{\prime} \sim 114^{\circ} 30^{\prime}$ & 2.0 & 32 \\
\hline
\end{tabular}

Table 2 Distribution range of the selected stations in the earthquake cluster zones

\begin{tabular}{clcc}
\hline No. & \multicolumn{1}{c}{ Zone } & $\varphi_{\mathrm{N}}$ & $\lambda_{\mathrm{E}}$ \\
\hline 1 & Wen'an & $37^{\circ} 30^{\prime} \sim 40^{\circ} 00^{\prime}$ & $115^{\circ} 00^{\prime} \sim 118^{\circ} 30^{\prime}$ \\
2 & Beijing & $38^{\circ} 30^{\prime} \sim 41^{\circ} 00^{\prime}$ & $116^{\circ} 00^{\prime} \sim 118^{\circ} 30^{\prime}$ \\
3 & Beijing-Tianjin & $38^{\circ} 30^{\prime} \sim 41^{\circ} 30^{\prime}$ & $116^{\circ} 00^{\prime} \sim 118^{\circ} 30^{\prime}$ \\
4 & Tangshan & $39^{\circ} 00^{\prime} \sim 41^{\circ} 30^{\prime}$ & $116^{\circ} 30^{\prime} \sim 119^{\circ} 30^{\prime}$ \\
5 & Luanxian & $39^{\circ} 30^{\prime} \sim 41^{\circ} 30^{\prime}$ & $117^{\circ} 00^{\prime} \sim 119^{\circ} 30^{\prime}$ \\
6 & Xingtai & $37^{\circ} 00^{\prime} \sim 39^{\circ} 00^{\prime}$ & $113^{\circ} 00^{\prime} \sim 118^{\circ} 00^{\prime}$ \\
7 & NW of Beijing & $39^{\circ} 00^{\prime} \sim 42^{\circ} 00^{\prime}$ & $113^{\circ} 00^{\prime} \sim 117^{\circ} 00^{\prime}$ \\
8 & SW of Beijing & $38^{\circ} 00^{\prime} \sim 41^{\circ} 00^{\prime}$ & $113^{\circ} 30^{\prime} \sim 117^{\circ} 00^{\prime}$ \\
\hline
\end{tabular}

\subsection{Restrictions on the precision of velocity calculation}

1) The number of seismic stations involved in the computation for each event (only $\mathrm{P}_{\mathrm{G}}$ and $\mathrm{S}_{\mathrm{G}}$ data are used) should be no smaller than five and most satisfactorily larger than eight; standard deviation of $v_{\mathrm{P}} / v_{\mathrm{S}}: S \leq 1.0$; correlation coefficient of $v_{\mathrm{P}} / v_{\mathrm{S}}: R_{1} \geq 0.95$; correlation coefficient of $v_{\mathrm{P}}: R_{2} \geq 0.95$;

2) For the zones with densely distributed stations (Beijing, Beijing-Tianjin, Wen'an, Tangshan, Luanxian and Xingtai), $t_{\mathrm{S}}-t_{\mathrm{P}}$ within $0 \sim 20 \mathrm{~s}$; for those with relatively sparse stations (northwest of Beijing, southwest of Beijing), we chose $t_{\mathrm{S}}-t_{\mathrm{P}}$ within $0 \sim 25 \mathrm{~s}$, so as to make sure that a certain number of earthquakes are involved in the study;

3) Besides the above restrictions, the ranges for the selection of stations in Beijing and Beijing-Tianjin, which are close to Wen'an, are restricted to a common area of $38^{\circ} 30^{\prime} \mathrm{N} \sim 41^{\circ} 30^{\prime} \mathrm{N}$, $116^{\circ} 00^{\prime} \mathrm{E} \sim 118^{\circ} 30^{\prime} \mathrm{E}$, while the ranges for other zones are different from each other, so as to identify similarities and differences in velocity ratio between different zones. The spatial ranges of the selected stations in the studied zones are shown in Table 2.

\section{Stability and reliability analysis of the result}

\subsection{The effect of combined use of borehole a nd bedrock data on the stability of the results}

In view of the difference in observation condition between bedrock stations and borehole stations, only the arrival time data from bedrock stations were used in the earlier stage of wave velocity computation. This has reduced the impact of site effect, geometric spreading and media absorption to some extent. However, borehole stations account for 50\% (53/107) of the 107 stations 
in the Capital Circle area (Figure 2), therefore large amounts of arrival data could not be put to full use. This had a negative effect especially on the case study of Wen'an earthquake. Borehole stations are distributed in plain areas while bedrock stations are in mountainous regions, hence the use of bedrock data alone could not convincingly define property variation of subsurface media in the plain area near Wen'an. Since seismic recording became digitized, experienced researchers could magnify the waveform for precise identification, and the precision could usually be as high as $0.02 \mathrm{~s}$ or so. Therefore, we examined carefully the identification precision of borehole data in the Capital Circle area and compared it with that of bedrock data. The result shows that, with numerous data from bedrock stations as restraints, the identification precision of arrival time data from borehole stations could also reach $0.02 \mathrm{~s}$ or so. Let's take Beijing-Tianjin and Luanxian seismic zones for example (Tables 3 and 4). After analytical comparison of mean standard errors of the results yielded respectively with bedrock data, borehole data and integrated bedrock-borehole data, we found that the results derived from $\geq 99 \%$ bedrock data and with $\geq 99 \%$ borehole data have approximately the same mean standard error, with a difference as small as $1 \sim 2 \%$. Comparison between mean standard errors derived from respective use and combined use of the two types of data demonstrates that the mean standard errors are also very close to each other and are stable as well. This indicates that the difference in station background is not the major factor affecting linear fitting deviation. As can be seen from Tables 2 and 3, the combined use of bedrock and borehole data obviously increased the number of stations involved in the computation, thus raising utilization ratio of natural earthquakes under condition of stable mean standard error (Tables 3 and 4). This result is extremely important for improving velocity precision of the areas under surveillance.

Table 3 Comparison between mean standard errors of $v_{\mathrm{P}} / v_{\mathrm{S}}$ in Beijing-Tianjin obtained by using bedrock and borehole data respectively and jointly

\begin{tabular}{lccc}
\hline Beijing-Tianjin $(N=103)$ & Mainly bedrock & Mainly borehole & Bedrock + Borehole \\
\hline Mean standard error $S$ & 0.56 & 0.57 & 0.59 \\
Number of qualified shocks & 74 & 38 & 81 \\
Utilization ratio of shocks & $72 \%$ & $37 \%$ & $79 \%$ \\
\hline
\end{tabular}

Table 4 Comparison between mean standard errors of $v_{\mathrm{P}} / v_{\mathrm{S}}$ in Luanxian obtained by using bedrock and borehole data respectively and jointly

\begin{tabular}{lccc}
\hline Luanxian $(N=112)$ & Mainly bedrock & Mainly borehole & Bedrock+Borehole \\
\hline Mean standard error $S$ & 0.59 & 0.61 & 0.58 \\
Number of qualified shocks & 61 & 25 & 83 \\
Utilization ratio of shocks & $59 \%$ & $22 \%$ & $74 \%$ \\
\hline
\end{tabular}

The above results indicates that borehole data and bedrock data differ very little in terms of identification precision, and there is no obvious difference between the calculation results yielded by respective use and combined use of the two types of data. Statistical results of seismic velocity data show that $79 \%$ of the selected earthquakes which satisfy the restrictions have arrival time data from eight seismic stations and $70 \%$ have data from 10 (Figure 3), i.e. most of the earthquakes have arrival time data from at least 10 stations, some even from as many as 48 . In addition, statistical analysis of the relationship between velocity result and the number of stations also show that velocity error is closely related to the number of stations: the larger the number of stations, the smaller the error, and fitting of arrival time data from more than 10 stations can reduce the error 
by half (Figure 4). In Luanxian, for example, when $t_{\mathrm{S}}-t_{\mathrm{P}} \leq 20 \mathrm{~s}$, the number of stations involved in fitting for a single event can only reach 12 if bedrock data are used alone, whereas the number can be as large as 22 if bedrock and borehole data are used jointly, which effectively improves the precision in velocity determination. In practical computation, when the arrival time data taken from at least 10 stations are involved in the fitting, the seismic sample size and temporal coverage can still be guaranteed at a certain degree in some of the zones which have relatively precise seismic records and dense stations, such as Beijing, Beijing-Tianjin and Luanxian. The number of stations involved in data fitting can be no smaller than 5 in Wen'an, northwest of Beijing, southwest of Beijing and Xingtai where seismic stations are relatively sparsely distributed. The abundant phase data which satisfy the restrictions and stable identification precision provide ideal conditions for enhancing objectivity of $v_{\mathrm{p}} / v_{\mathrm{s}}$ and wave velocity results.

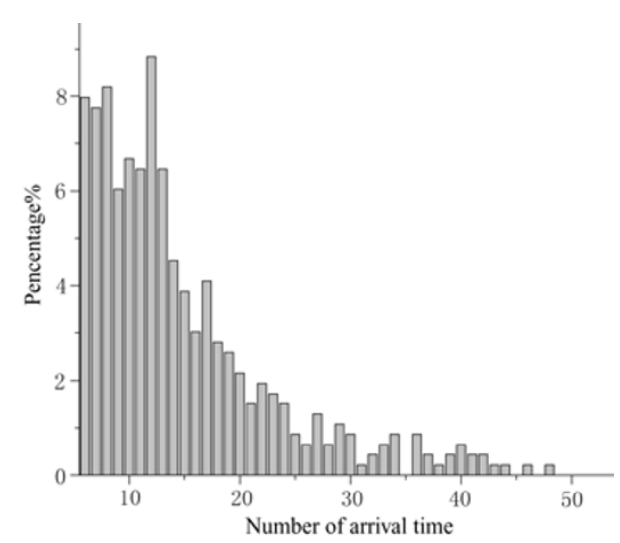

Figure 3 Statistical distribution of the number of stations for each selected earthquake

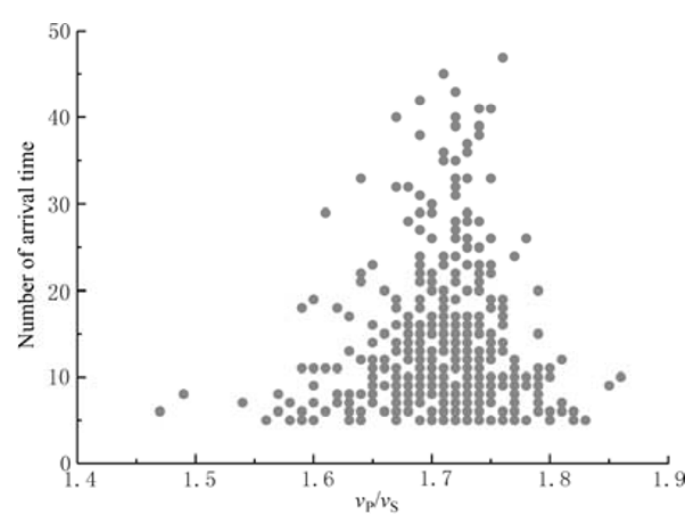

Figure 4 Relation between $v_{\mathrm{P}} / v_{\mathrm{S}}$ value and the number of stations involved in the computation

\subsection{Stability and error analysis on the results of $v_{\mathrm{P}} / v_{\mathrm{S}}$ and $v_{\mathrm{P}}$}

$v_{\mathrm{P}} / v_{\mathrm{S}}$ value $\gamma$ is derived from equation (4), and one important step for its reliability analysis is error analysis. Linear correlation coefficient $R_{1}$ of $v_{\mathrm{P}} / v_{\mathrm{S}}$ is derived from equation (5), and standard deviation $S$ of $v_{\mathrm{P}} / v_{\mathrm{S}}$ is derived from equation (8), where $t_{\mathrm{P} i}$ denotes $\mathrm{P}$-wave arrival time, $t_{\mathrm{S}}-t_{\mathrm{P}}$ denotes arrival time difference between $\mathrm{S}$ and $\mathrm{P}$ wave, and $n$ represents the number of stations. Error analysis and correlation coefficient analysis reveal that $S \leq 1.0$ is an adequate choice for securing relatively small error of $v_{\mathrm{P}} / v_{\mathrm{S}}$ (ZHAO et al, 2005).

$\mathrm{P}$-wave velocity $v_{\mathrm{P}}$ is obtained from equation (6) and its linear correlation $R_{2}$ is obtained from equation (7), where $t_{\mathrm{P} i}$ denotes P-wave arrival time, $D_{i}$ source-to-station distance, and $n$ the number of stations.

$$
\frac{v_{\mathrm{P}}}{v_{\mathrm{S}}}=1+\frac{n \sum_{i=1}^{n}\left(t_{\mathrm{S}}-t_{\mathrm{P}}\right)^{2}-\left(\sum_{i=1}^{n}\left(t_{\mathrm{S}}-t_{\mathrm{P}}\right)\right)^{2}}{n \sum_{i=1}^{n}\left(t_{\mathrm{S}}-t_{\mathrm{P}}\right) t_{\mathrm{P} i}-\sum_{i=1}^{n} t_{\mathrm{P} i}\left(t_{\mathrm{S}}-t_{\mathrm{P}}\right)}
$$




$$
\begin{aligned}
& R_{1}=\frac{n \sum\left(t_{\mathrm{P} i}-\overline{t_{\mathrm{P} i}}\right)\left[\left(t_{\mathrm{S}}-t_{\mathrm{P}}\right)-\overline{\left(t_{\mathrm{S}}-t_{\mathrm{P}}\right)}\right]}{\sqrt{\left.\sum_{i=1}^{n}\left(t_{\mathrm{P} i}-\overline{t_{\mathrm{P} i}}\right)^{2} \sum_{i=1}^{n}\left[\left(t_{\mathrm{S}}-t_{\mathrm{P}}\right)-\overline{\left(t_{\mathrm{S}}-t_{\mathrm{P}}\right.}\right)\right]^{2}}} \\
& v_{\mathrm{P}}=\frac{n \sum_{i=1}^{n} D_{i}^{2}-\left(\sum_{i=1}^{n} D_{i}\right)^{2}}{n \sum_{i=1}^{n} D_{i} t_{\mathrm{P} i}-\sum_{i=1}^{n} t_{\mathrm{P} i} D_{i}} \\
& R_{2}=\frac{n \sum_{i=1}^{n}\left(t_{\mathrm{P} i}-\overline{t_{\mathrm{P} i}}\right)\left(D_{i}-\overline{D_{i}}\right)}{\sqrt{\sum_{i=1}^{n}\left(t_{\mathrm{P} i}-\overline{t_{\mathrm{P} i}}\right)^{2} \sum_{i=1}^{n}\left(D_{i}-\overline{D_{i}}\right)^{2}}} \\
& \left.S=\sqrt{\frac{1}{n(n-2)}\left\{n \sum_{i=1}^{n} t_{\mathrm{P} i}^{2}-\left(\sum_{i=1}^{n} t_{\mathrm{P} i}\right)^{2}-\frac{\left\{n \sum_{i=1}^{n}\left(t_{\mathrm{S}}-t_{\mathrm{P}}\right) t_{\mathrm{P} i}-\left[\sum_{i=1}^{n}\left(t_{\mathrm{S}}-t_{\mathrm{P}}\right)\right] \sum_{i=1}^{n} t_{\mathrm{P} i}\right\}^{2}}{n \sum_{i=1}^{n}\left(t_{\mathrm{S}}-t_{\mathrm{P}}\right)^{2}-\left[\sum_{i=1}^{n}\left(t_{\mathrm{S}}-t_{\mathrm{P}}\right)\right]^{2}}\right.}\right\}
\end{aligned}
$$

Here, $\Delta \bar{t}=\frac{1}{n} \sum_{i=1}^{n}\left(t_{\mathrm{S}}-t_{\mathrm{P}}\right), \Delta \bar{T}_{P}=\frac{1}{n} \sum_{i=1}^{n} \Delta T_{\mathrm{P} i}$ denote respectively arithmetic means of $t_{\mathrm{S}}-t_{\mathrm{P}}$ and $\mathrm{P}$ arrival time.

To examine stability of the result, we made a statistical analysis of 464 earthquakes in the eight studied zones which satisfy the above restrictions (arrival time data from at least five stations, $R_{1} \geq 0.95, R_{2} \geq 0.95$ and $S \leq 1.0$ ), and carried out a systematic investigation on the distribution of their $v_{\mathrm{P}} / v_{\mathrm{S}}$ and $v_{\mathrm{P}}$ values. Figure 5 presents $v_{\mathrm{P}} / v_{\mathrm{S}}$ and $v_{\mathrm{P}}$ values versus the number of earthquakes, from which we can see that $v_{\mathrm{P}} / v_{\mathrm{S}}$ and $v_{\mathrm{P}}$ values are both highly concentrated around the mean values. For $v_{\mathrm{P}} / v_{\mathrm{S}}$, the mean values 1.71 and $1.6 \sim 1.8$ account for $94 \%$, and $1.4 \sim 1.9$ account for $99.6 \%$; for $v_{\mathrm{P}}$, the mean values 5.93 and 5.5 6.5 account for $92 \%$. Figure 6 is a statistical graph showing standard deviation $S$ of $v_{\mathrm{P}} / v_{\mathrm{S}}$ versus the number of earthquakes. Standard deviation $S$ of $v_{\mathrm{P}} / v_{\mathrm{S}}$ is 0.58 and $S \leq 0.8$ makes up $82 \%$, while $S \leq 0.9$ makes up $92 \%$. Figure 7 shows the correlation coeffi-
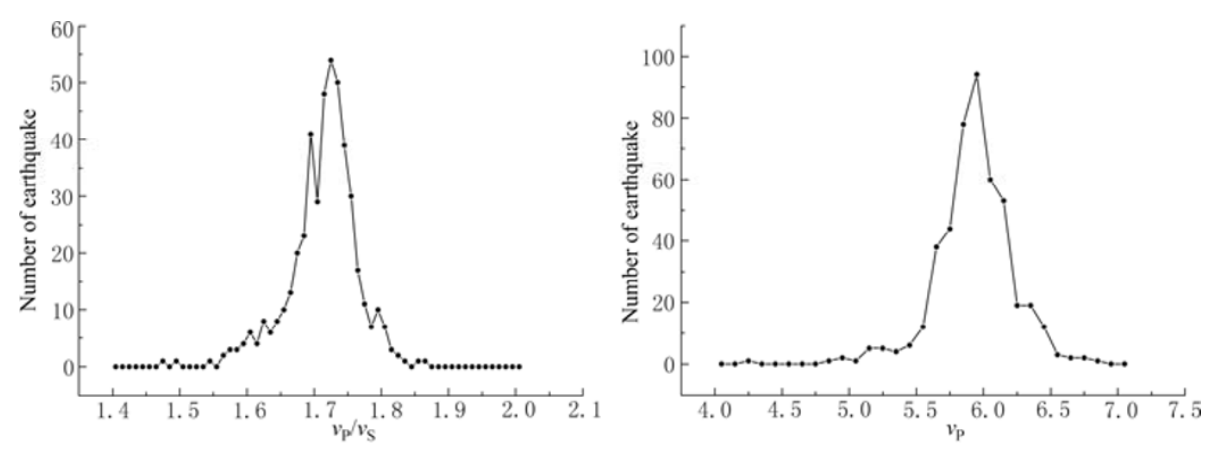

Figure $5 \quad v_{\mathrm{P}} / v_{\mathrm{S}}(\mathrm{a})$ and $v_{\mathrm{P}}(\mathrm{b})$ versus the number of earthquakes 
cients $R_{1}$ of $v_{\mathrm{P}} / v_{\mathrm{S}}$ and $R_{2}$ of $v_{\mathrm{P}}$ corresponding to the number of earthquakes. Distribution ratio of the correlation coefficients $R_{1}$ and $R_{2}$ is shown in Table 5, where we can see that the ratio of $R_{1}>0.99$ reaches $74 \%$, much lower than that of $R_{2}$ which is $98 \%$. Table 5 also indicates that $\mathrm{P}$-wave velocity calculation depends mainly on P-wave arrival time and source-to-station distance. In spite of the

Table 5 Comparison between correlation coefficients $R_{1}$ of $v_{\mathrm{P}} / v_{\mathrm{S}}$ and $R_{2}$ of $v_{\mathrm{P}}$

\begin{tabular}{ccc}
\hline Corelation coefficient & $\geq 0.98$ & $\geq 0.99$ \\
\hline$R_{1}$ & $90 \%$ & $74 \%$ \\
$R_{2}$ & $99 \%$ & $98 \%$ \\
\hline
\end{tabular}

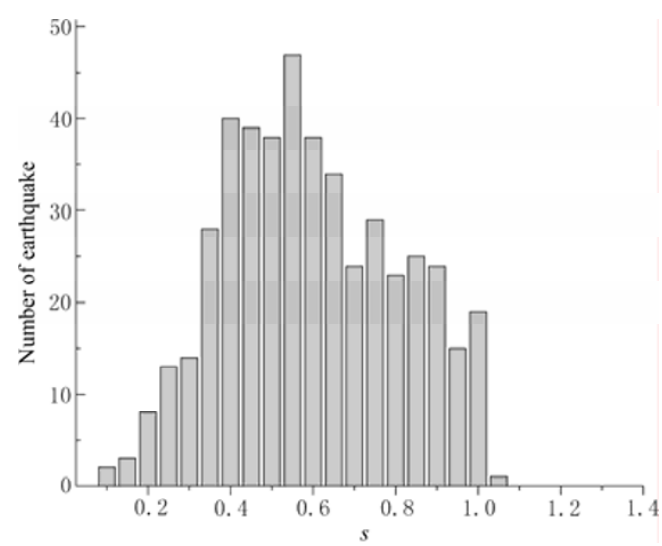

Figure 6 Standard deviation $S$ of $v_{\mathrm{P}} / v_{\mathrm{S}}$ versus the number of earthquakes

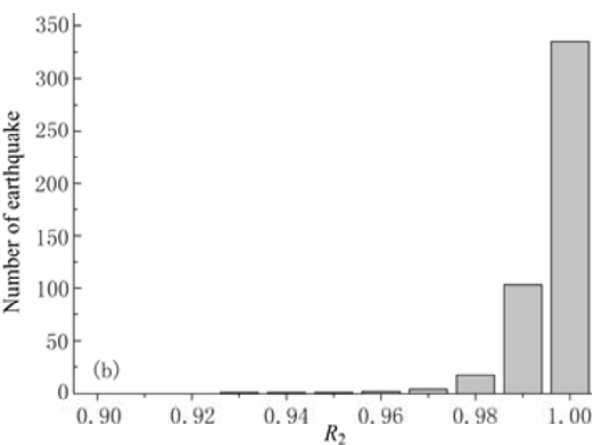

Figure 7 Correlation coefficients $R_{1}$ of $v_{\mathrm{P}} / v_{\mathrm{S}}$ (a) and $R_{2}$ of $v_{\mathrm{P}}$ (b) corresponding to the number of earthquakes

errors in source location (particularly depth location), the precision of location has been greatly improved for the earthquakes occurring in the inner part of the seismograph network, thanks to high density of the stations in the network. Therefore, the precision of P-wave velocity is higher than that of $v_{\mathrm{P}} / v_{\mathrm{S}}$, as judged by correlation coefficients of linear fitting.

\section{Results of different zones}

\subsection{Wen'an and the adjacent zones}

The distribution ranges of the earthquakes in Wen'an, Tangshan, Beijing-Tianjin and Beijing are listed in Table 1. For Wen'an, we listed the $M \geq 1.0$ events between January 2002 and April 2007, and for Tangshan, Beijing-Tianjin and Beijing we listed the $M \geq 1.0$ events between January 2002 and October 2007. Out of these events we chose 247 which have seismic data from more than five stations, and calculated $v_{\mathrm{P}} / v_{\mathrm{S}}$ values of each zone separately. We then examined $v_{\mathrm{P}} / v_{\mathrm{S}}$ variation characteristics for each zone before Wen'an earthquake. The temporal $v_{\mathrm{P}} / v_{\mathrm{S}}$ variations of the four zones are shown in Figure 8. Moving average method with five events is adopted so as to avoid fluctuation of single-event $v_{\mathrm{P}} / v_{\mathrm{S}}$ and thus, $v_{\mathrm{P}} / v_{\mathrm{S}}$ variation before the earthquake can be reflected more clearly. Under normal conditions, the moving average value of $v_{\mathrm{P}} / v_{\mathrm{S}}$ varies very little, 
but before Wen'an earthquake the velocity ratio varies sharply. As can be seen in Figure 8, the velocity ratio before the event became obviously low, with a difference of twice its standard deviation and the anomaly lasted for about one year. Meanwhile, little variation of $v_{\mathrm{P}} / v_{\mathrm{S}}$ was seen in Beijing-Tianjin and Beijing. After the event, the ratio soon returned normal in Wen'an and Tangshan. In brief, $v_{\mathrm{P}} / v_{\mathrm{S}}$ in and around Wen'an and Tangshan underwent a normal-low-normal process before Wen'an earthquake, and the anomaly faded gradually with increasing distance from the epicenter (Figure 8).
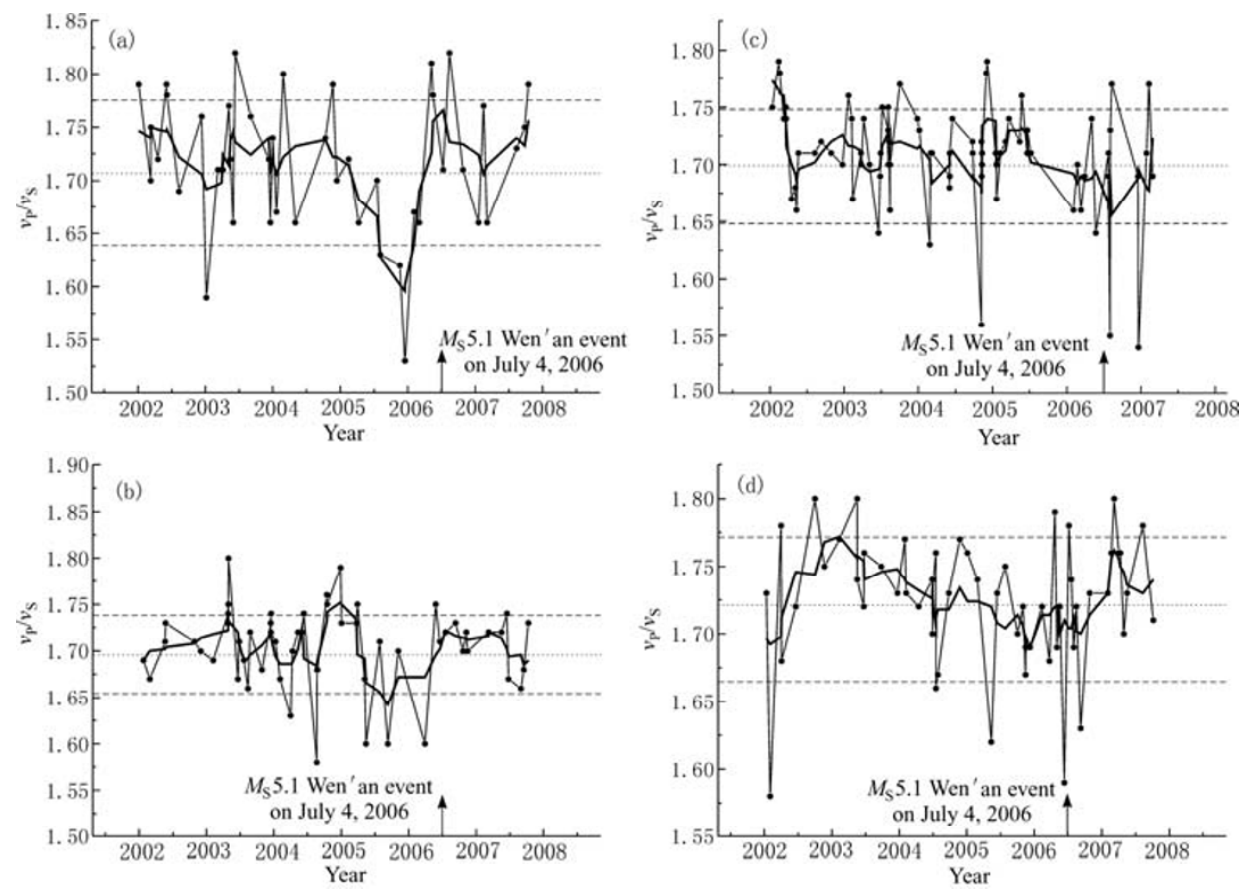

Figure $8 \quad v_{\mathrm{P}} / v_{\mathrm{S}}$ versus time curve in Wen'an (a), Tangshan (b), Beijing-Tianjin (c) and Beijing (d) Thick curve denotes moving average of five events; dotted line denotes the mean and dashed line denotes standard deviation

Figure 9 displays the coverage of seismic rays in these four zones. In Wen'an, seismic rays are most densely distributed and anomaly of low $v_{\mathrm{P}} / v_{\mathrm{S}}$ is the most obvious in the seismogenic area of Wen'an earthquake; in Tangshan, which is a historical earthquake region where densely distributed earthquakes occurred frequently, seismic rays are densely distributed close to the northeast of the seismogenic area of Wen'an earthquake. This is the reason why anomaly of the velocity ratio is more obvious in Tangshan than in Beijing-Tianjin and Beijing. Judging by the characteristics of the velocity ratio in the above 4 zones, the anomalous range of low $v_{\mathrm{P}} / v_{\mathrm{S}}$ for Wen'an earthquake is within a radius of about $100 \mathrm{~km}$.

\subsection{West and south of the Capital Circle area}

The above analysis demonstrates temporal and spatial variations of $v_{\mathrm{P}} / v_{\mathrm{S}}$ anomaly relative to Wen'an earthquake. In order to probe further into the spatial distribution characteristics of $v_{\mathrm{P}} / v_{\mathrm{S}}$ anomaly relevant to the earthquake, we respectively examined $v_{\mathrm{P}} / v_{\mathrm{S}}$ variations in other zones associated with the Capital Circle area. These mainly include the zones northwest of Beijing, southwest of Beijing and Xingtai (see Table 1 for the ranges of selected earthquakes). The earthquakes in 

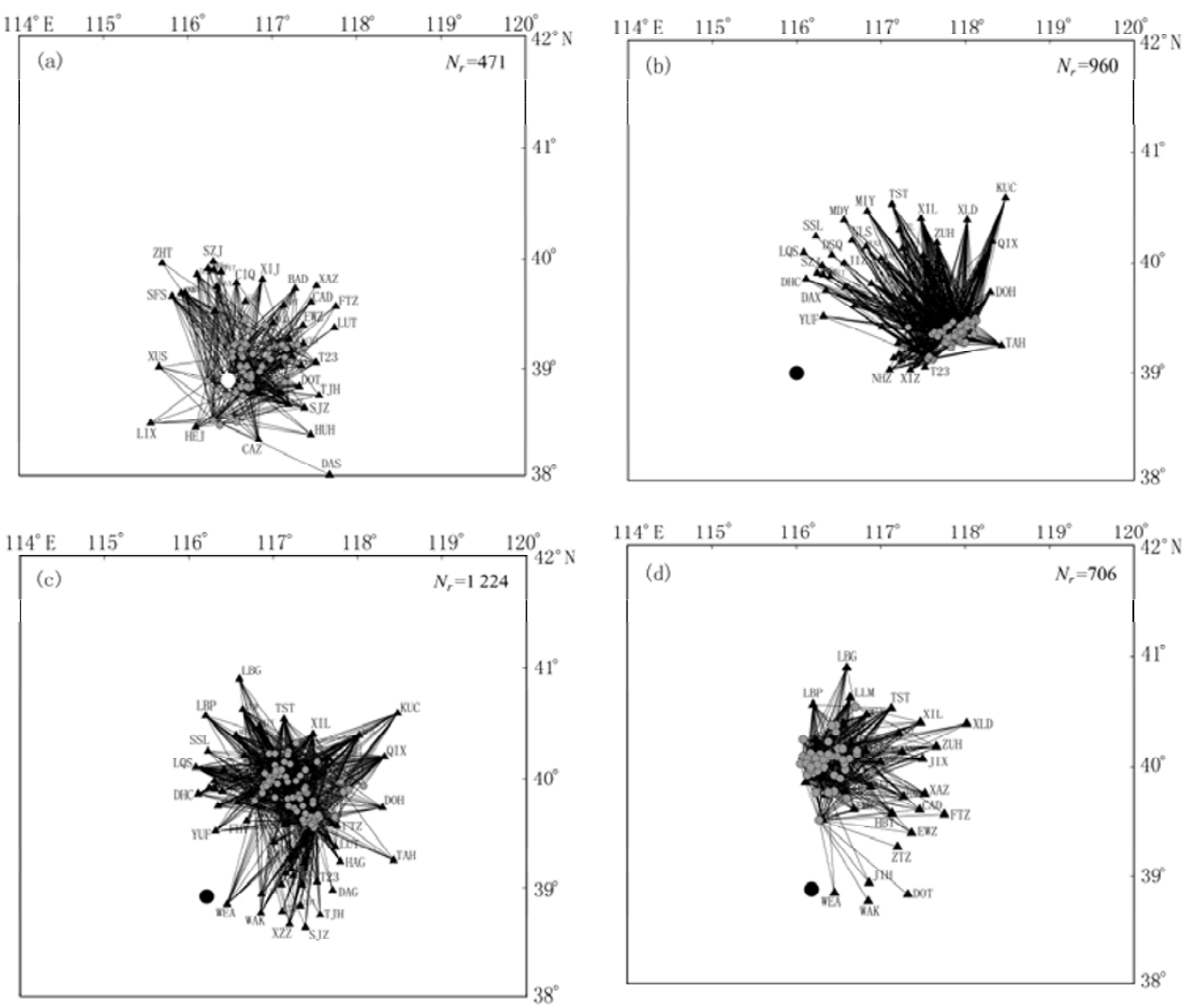

Figure 9 Ray spatial distribution in Wen'an (a), Tangshan (b), Beijing-Tianjin (c) and Beijing (d) $N_{r}$ represents the total number of rays

these zones, with magnitude of $M_{\mathrm{L}} \geq 2$, correspond with those of the above four zones in time range.

Figure 10 displays $v_{\mathrm{p}} / v_{\mathrm{S}}$ versus time curve of the three zones respectively. It shows that in the above three zones, moving average of $v_{\mathrm{p}} / v_{\mathrm{S}}$ with equal events since 2002 varies very little and no obvious anomaly of the velocity ratio is seen before Wen'an earthquake. This indicates that the anomaly of $v_{\mathrm{P}} / v_{\mathrm{S}}$ ratio appears mainly in the vicinity of Wen'an and Tangshan, featuring spatial regionality. The spatial range of $v_{\mathrm{p}} / v_{\mathrm{S}}$ anomaly for the Wen'an earthquake might be a little too large relative to its magnitude $\left(M_{\mathrm{S}} 5.1\right)$, which is supposed to be attributed to limited density of seismic stations in the Capital Circle area and adequate number of seismic stations required by Wadati method.

\section{Discussion and conclusions}

1) In order to find out if $v_{\mathrm{P}}$ decreases more distinctly than $v_{\mathrm{S}}$ in the anomaly of low $v_{\mathrm{P}} / v_{\mathrm{S}}$, we divided the earthquakes in the obviously abnormal Wen'an and Tangshan zones into normal-phase events and abnormal-phase events according to the phase when anomalously low values emerge. We separately calculated the general average velocity of $v_{\mathrm{P}}$ and $v_{\mathrm{S}}$ based on all the phase data involved in the computation and identified similarities and differences between general $v_{\mathrm{P}}$ and $v_{\mathrm{S}}$ velocities in the two different phases. Since they are general velocities, the number of arrival time data involved in the computation should respectively reach 54 and 65 in the abnormal phase, and 

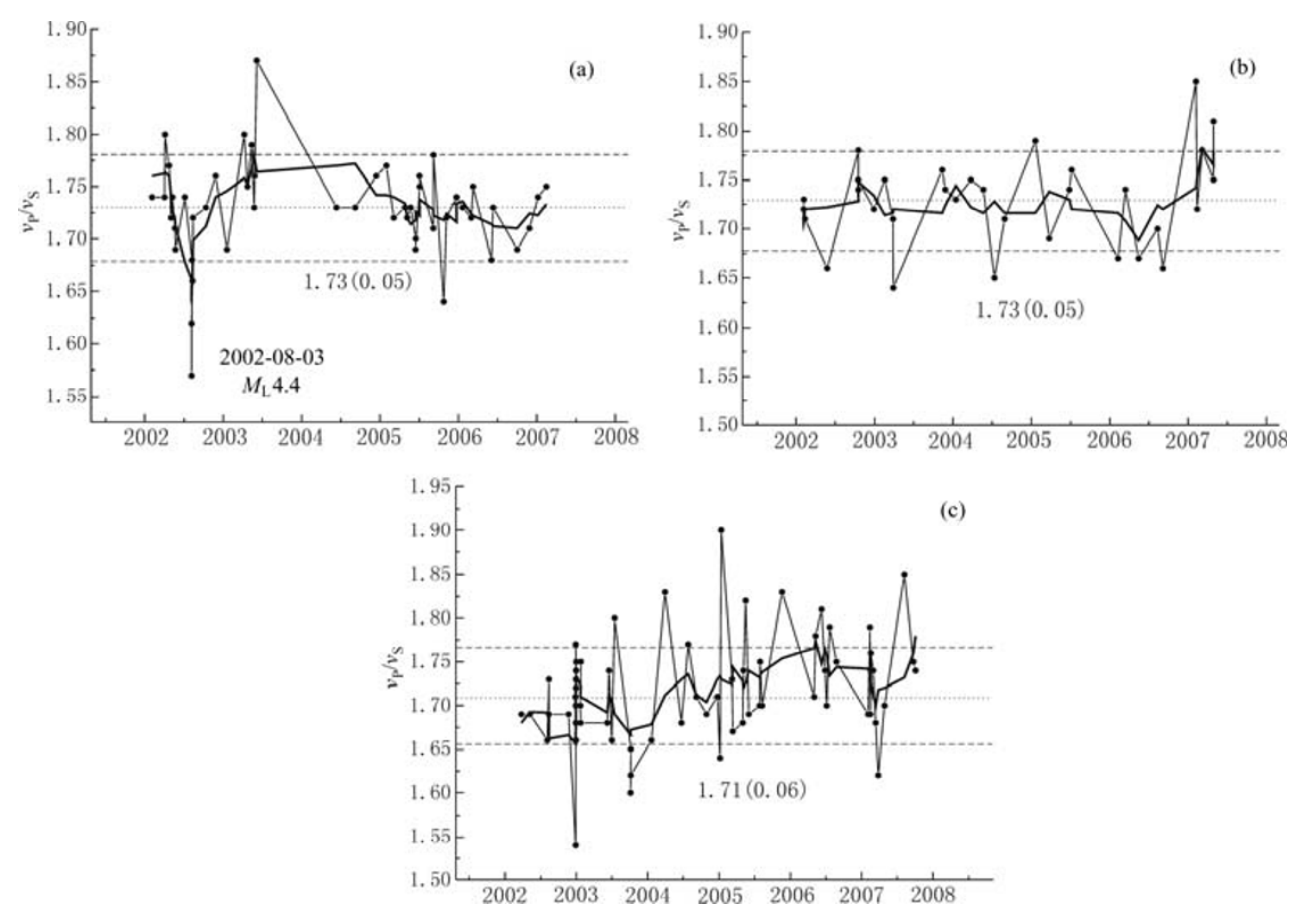

Figure $10 v_{\mathrm{p}} / v_{\mathrm{S}}$ versus time curve in northwest of Beijing (a), southwest of Beijing (b) and Xingtai (c) Red curve denotes moving average of 5 events; dotted line denotes the mean and dashed line denotes the standard deviation

453 and 975 in the normal phase so as to secure high precision and stability of the results. Table 6 presents a comparison between general $v_{\mathrm{P}}$ and $v_{\mathrm{S}}$ velocities in normal phase and those in abnormal phase. It shows that the general $v_{\mathrm{P}}$ value in Wen'an and Tangshan is obviously lower in the abnormal phase than in normal one, with an average difference of 0.191 , which is $3 \%(0.191 / 5.957)$ of the average normal $v_{\mathrm{P}}$, whereas, the average $v_{\mathrm{S}}$ value in abnormal phase differs little from that in the normal one: it is only 0.020 lower, which is $0.6 \%(0.020 / 3.474)$ of the normal $v_{\mathrm{S}}$ value. On the whole, $v_{\mathrm{P}}$ value varies 5 times as much as $v_{\mathrm{S}}$ does. This suggests that $v_{\mathrm{P}}$ changes more greatly than $v_{\mathrm{S}}$ in the variation of $v_{\mathrm{P}} / v_{\mathrm{S}}$ and the abnormal low $v_{\mathrm{P}} / v_{\mathrm{S}}$ before Wen'an earthquake was attributed to low $v_{\mathrm{P}}$ value. The linear correlation coefficients of the above results are all above 0.992 . In Table 6, the $n$ in brackets stands for the total number of phases. ZHAO et al (2005) also concluded that $\mathrm{P}$ wave often reflects volume deformation characteristics of the media while $\mathrm{S}$ wave mainly reflects shear deformation. This indicates that volume elasticity change of the media near the hypocenter before Wen'an earthquake is greater than shear deformation, and it is most probably related with the rapid development of microcracks in and around the focus region before the shock.

In Figure 8, we can see an obvious normal-low-normal process of $v_{\mathrm{p}} / v_{\mathrm{S}}$ ratio in and around Wen'an and Tangshan before Wen'an earthquake. According to the experiment of rock sample failure, wave velocity increases with increasing pressure in the initial stage, but decreases obviously with increasing pressure as the pressure exceeds $50 \%$ of rock strength before rock failure. The phenomenon can be accounted for by the emergence of microcracks due to structural change, followed by cubic dilatancy (FENG, 1981). The velocity variations before the quake can be explained by Nur's dilatancy model (Nur, 1972), which deems that in the abnormal phase of wave 
velocity, microcracks occur in succession when stress exceeds one half of the rock strength. Under dilatancy effect, wave velocity begins to decrease and after reaching the lowest point, it remains stable for a period of time, which is considered to be a seismically quiescent period. This is because dilatancy reduces pore pressure and actually enhances the effective rock failure strength, thus preventing earthquakes from happening. With the infiltration of external ground water, the edema effect enhances pore pressure, then elastic modulus of rocks becomes larger and wave velocity rises. As the infiltration process is slow, it takes a certain period of time for the velocity to return to normal, which is the period for earthquake occurrence (LI, 1981). The abnormal process of $v_{\mathrm{P}} / v_{\mathrm{S}}$ before the quake revealed with Wadati method reflects the variation characteristics of the upper crust media. Wen'an earthquake took place in the depressed northern part of North China, where crustal thickness is small. To find out if geothermal behavior occurs in the deep focus region, velocity calculation methods with other phases or with $Q$ factor are needed to analyze property variation of deeper media before the quake, so that a more objective and overall explanation could be given for the mechanism of difference between $v_{\mathrm{P}}$ and $v_{\mathrm{S}}$ velocity variations.

Table 6 Comparison between velocities of $v_{\mathrm{P}}$ and $v_{\mathrm{S}}$ in normal phase and those in abnormal phase

\begin{tabular}{clllll}
\hline \multirow{2}{*}{ Zone } & \multicolumn{2}{c}{$v_{\mathrm{P}}$} & & & $v_{\mathrm{S}}$ \\
\cline { 2 - 3 } \cline { 5 - 6 } & Normal phase & Abnormal phase & & Normal phase & Abnormal phase \\
\hline Wen'an & $5.942(n=453)$ & $5.793(n=54)$ & & $3.467(n=453)$ & $3.487(n=54)$ \\
Tangshan & $5.972(n=904)$ & $5.738(n=59)$ & & $3.480(n=904)$ & $3.420(n=59)$ \\
Average & 5.957 & 5.766 & 3.474 & 3.454 \\
\hline
\end{tabular}

2) Although Wen'an earthquake has a magnitude of only $M_{\mathrm{S}} 5.1$, the area of its $v_{\mathrm{P}} / v_{\mathrm{S}}$ variation (with a radius of some $100 \mathrm{~km}$ around the epicenter) is large relative to the range of its focus region. Then what can account for the large-scale variation? In this study, we summarize the reasons as follows:

Firstly, the method used for wave velocity calculation is the Wadati method. FENG (1981) stressed that wave velocity anomaly can be discovered by variations of curvature of the Wadati diagrams, provided that seismic stations are densely distributed and minor earthquake sources located in or around the anomalous area. However, it must be pointed out that the average $v_{\mathrm{P}} / v_{\mathrm{S}}$ estimated by the slope in Wadati diagram is not genuine $v_{\mathrm{P}} / v_{\mathrm{S}}$, instead, it is only apparent $v_{\mathrm{P}} / v_{\mathrm{S}}$. Therefore, in case of monolayer homogeneous media, the average result of linear fitting with multi-station seismic data depends mainly on P- and S-wave arrival time data, and the result provides comprehensive information about property variation of the media from above the hypocenter to the ground surface, which is somewhat complex and multiplex. Further study is needed to find out if there is any difference in physical property between the anomalous range of the uppermost crustal stratum and that of the deeper focus region. To insure precision of the result, the number of seismic stations involved in computation for each event should exceed 5 and $80 \%$ of the events should have seismic data from at least 8 . Wen'an earthquake took place in the south of the capital circle area seismograph network, where station gap is roughly as large as $100 \mathrm{~km}$ and spatial resolution is obviously low, thus, it is difficult to identify more precisely velocity variation within a small range of tens of kilometers. This study only provides a very rough estimation about the range of $v_{\mathrm{P}} / v_{\mathrm{S}}$ anomaly.

Secondly, the basic range of $v_{\mathrm{P}} / v_{\mathrm{S}}$ anomaly for most of the $M_{\mathrm{S}} 5$ earthquakes in western China has a long radius of 50 60 km and a short radius of 20 30 km. In foreign literature, some scholars argue that the range of $v_{\mathrm{P}} / v_{\mathrm{S}}$ anomaly is $2 \sim 3$ times as large as the focus region, some argue it is 8 
(FENG, 1981), suggesting a large discrepancy. Wen'an is located in the south center of the capital circle area, where crustal thickness is small and NW- and NE-oriented structures intertwine with one another. The complexity of geologic setting and stress accumulation environment and peculiarity of seismogenic mechanism might be major factors responsible for the large range of $v_{\mathrm{P}} / v_{\mathrm{S}}$ variations.

The above analysis reveals that there are still many issues to be studied concerning tectonic environment and seismogenic mechanism of Wen'an earthquake.

3) The low utilization ratio of borehole data relative to bedrock data is attributed to its higher background noise, minor displacement, earlier P-wave arrival time and higher frequency, all of which make it difficult to unify data interpretation. In case of precise earthquake location, by restraining the identification precision of borehole data with bedrock data (which is a very important step in phase identification), the identification precision of borehole data could be greatly improved, the number of stations available for $v_{\mathrm{p}} / v_{\mathrm{S}}$ calculation become larger, and data distribution of earthquakes with different station-epicenter distances become more uniform and rational, thus, the combined use of borehole data and bedrock data could greatly help improve the precision of wave velocity calculation. In contrast, wave velocity calculation with borehole data alone could yield relatively few results which satisfy precision criterion, and this is one major reason for the low utilization ratio of borehole data.

4) Observing temporal variations of subsurface medium property is an important approach as well as a potential research field for the study of earthquake prediction. Estimation of seismic wave velocity and acquisition of information about subsurface media property variation is absolutely significant and essential for predicting physical parameters of moderate and strong earthquakes, especially for increasing earthquake-related subsurface information.

\section{References}

DIAO Gui-ling, ZHOU Shi-yong, LIU Jie, et al. 2005. Anomalies of mean $v_{\mathrm{P}} / v_{\mathrm{S}}$ ratio in the series of Xiuyan earthquake [J]. North China Earthquake Sciences, 23(4): 1-5 (in Chinese).

FENG De-yi, GU Jin-ping, WANG Zhou-yuan. 1978. Preliminary study of the velocity anomalies of seismic waves before and after some strong and moderate earthquakes in western China (III): Variations of curvature of the Wadati diagrams [J]. Acta Geophysica Sinica, 21(4): 84-97 (in Chinese).

FENG De-yi, TAN Ai-na, WANG Ke-fen. 1974. Velocity anomalies of seismic waves from near earthquakes and earthquake prediction [J]. Acta Geophysica Sinica, 17(2): 84-97 (in Chinese).

FENG De-yi. 1981. Seismic Wave Velocity Anomaly [M]. Beijing: Seismological Press: 84-97 (in Chinese).

FENG Rui, PANG Qing-yan, FU Zheng-xiang. 1976. Variations of $v_{\mathrm{P}} / v_{\mathrm{S}}$ before and after the Haicheng earthquake of 1975 [J]. Acta Geophysica Sinica, 19(4): 84-97 (in Chinese).

Hauksson E and Haase J S. 1994. Three-dimensional $v_{\mathrm{P}} / v_{\mathrm{S}}$ velocity model of the Los Angeles Basin and central Transverse Ranges, California [J]. J Geophys Res, 102: 5 423-5 453.

Kanamori H and Fuis G. 1976. Variation of P wave velocity before and after the Galway Lake earthquake $\left(M_{\mathrm{L}}=5.2\right)$ and Goat Mountain earthquake $\left(M_{\mathrm{L}}=4.7\right)$, in the Mojave desert, California [J]. Bull Seism Soc Amer, 66: 2 027-2 037.

Korneev V A, McEvilly T V, Karageorgi E D. 2000. Seismological studies at Parkfield VIII: Modeling the observed travel-time changes [J]. Bull Seism Soc Amer, 90: 702-708.

LI Ming-xiao and LIU Jie. 2006. Study on velocity ratio $\left(v_{\mathrm{P}} / v_{\mathrm{S}}\right)$ anomaly of earthquake sequences in Yunnan region [J]. Earthquake, 26(1): 26-34 (in Chinese).

LI Ming-xiao and ZHANG Xiao-dong. 2004. Determining average seismic velocity ratios $\left(v_{\mathrm{P}} / v_{\mathrm{S}}\right)$ in the curst in North China region by multi-station method [J]. Earthquake, 24(1): 163-169 (in Chinese).

LI Shan-bang. 1981. Earthquakes in China [M]. Beijing: Seismological Press: 522-528, 566-575.

LI Y-G, Vidale J E, Aki K, et al. 1998. Evidence of shallow fault zone healing after the 1992 M7.5 Landers, California, earthquake [J]. Science, 279: 217-219.

McEvilly T V and Johnson L R. 1974. Stability of P and S velocities from central California quarry blasts [J]. Bull Seism Soc Amer, 64: 343-353.

Nakamura A, Hasegawa A, Hirata N, et al. 2002. Temporal variations of seismic wave velocity associated with 1998 M6.1 Shizukuishi earthquake [J]. Pure Appl Geophys, 159: 1 183-1 204.

Nur A. 1972. Dilatancy pore fluids and premonitory variations of $t_{\mathrm{s}} / t_{\mathrm{p}}$ travel times [J]. Bull Seism Soc Amer, 62: $1217-1222$. 
Schaff D P and Beroza G C. 2004. Coseismic and postseismic velocity changes measured by repeating earthquakes [J]. J Geophys Res, 109: B10302, doi:10.1029/2004JB003011.

Walck M C. 1988. Three-dimensional $v_{\mathrm{P}} / v_{\mathrm{S}}$ variations for the Coso region, California [J]. J Geophys Res, 93: 2 047-2 053.

ZHANG Ai-xiang. 2005. Study of the tempo-spatial evolution characteristics of velocity ratio before moderately strong earthquakes in Anyang and its adjacent regions [J]. J of College of Disaster Prevention Techniques, 7(1): 40-45 (in Chinese).

ZHANG Xiao-tao, LÜ Jian, MA Guang-qing, et al. 2006. Characteristics of $v_{\mathrm{P}} / v_{\mathrm{S}}$ ratio in the series of Jiujiang-Ruichang earthquake [J]. North China Earthquake Sciences, 24(4): 36-40 (in Chinese).

ZHANG Xue-min, DIAO Gui-ling, SHU Pei-yi. 2004a. Study on shear wave velocity structure and velocity ratio beneath Ordos block and its eastern and southern margins [J]. Earthquake Research in China, 20(1): 53-63 (in Chinese).

ZHANG Xue-min, DIAO Gui-ling, SHU Pei-yi. 2004b. Study on wave velocity ratio $\left(v_{\mathrm{P}} / v_{\mathrm{S}}\right)$ of underground media in North China [J]. Seismology and Geology, 26(2): 305-317 (in Chinese).

ZHANG Xue-min, ZHANG Tian-zhong, LI Yong-hong, et al. 2007. Study on multi-station seismic velocity ratios in Shandong area [J]. Seismology and Geology, 29(1): 95-104 (in Chinese)

ZHAO Ming-chun, DIAO Gui-ling, ZHANG Xue-min. 2005. Analysis on average velocity ratios in the capital area, China [J]. Northwestern Seismological Journal, 27(3): 245-254 (in Chinese).

ZOU Zhen-xuan, LI Jin-long, YU Tie-hong. 2006. Determining average seismic wave velocity ratios $\left(v_{\mathrm{P}} / v_{\mathrm{S}}\right)$ in Shanxi reservoir of Wenzhou region by using multi-station method [J]. Earthquake, 26(4): 133-137 (in Chinese) 